\title{
Congruence of selfadjoint operators
}

\author{
Guillermina Fongi and Alejandra Maestripieri
}

\begin{abstract}
Given a bounded selfadjoint operator $a$ in a Hilbert space $\mathcal{H}$, the aim of this paper is to study the orbit of $a$, i.e., the set of operators which are congruent to $a$. We establish some necessary and sufficient conditions for an operator to be in the orbit of $a$. Also, the orbit of a selfadjoint operator with closed range is provided with a structure of differential manifold.
\end{abstract}

Mathematics Subject Classification (2000). Primary 47B15, 58B20.

Keywords. Selfadjoint operators, congruence of operators, differential geometry.

\section{Introduction}

Let $L(\mathcal{H})$ be the algebra of linear bounded operators of a separable Hilbert space $\mathcal{H}$ and $G L(\mathcal{H})$ the group of invertible operators of $L(\mathcal{H})$. Two operators $a, b \in L(\mathcal{H})$ are congruent if there exists $g \in G L(\mathcal{H})$ such that $b=g a g^{*}$. If $a$ and $b$ are positive, then $a$ and $b$ are congruent if and only if their ranges $R(a)$ and $R(b)$ are unitarily equivalent, i.e. there exists a unitary operator $u$ such that $R(b)=u R(a)$, see [10]. In [11], it was proved that if $a$ and $b$ are invertible selfadjoint operators, $b$ is congruent to $a$ if and only if the reflections of their polar decompositions belong to the same unitary orbit. The purpose of this paper is to study the set of operators which are congruent with $a$, when $a$ is a given selfadjoint operator, not necessarily invertible.

The congruence between selfadjoint operators defines a natural action of the group $G L(\mathcal{H})$ over the set $L(\mathcal{H})^{s}$ of selfadjoint operators given by $L_{g} a=g a g^{*}$ and the set $L(\mathcal{H})^{s}$ is the (disjoint) union of the orbits of this action. In [8], Corach et al. described the set $G L(\mathcal{H})^{s}$, of selfadjoint invertible operators, as a reductive homogeneous space of $G L(\mathcal{H})$, with a canonical connection and a Finsler metric. They proved that any pair of points $a, b \in G L(\mathcal{H})^{s}$ having the same unitary part in the polar decomposition, can be joined by a geodesic. These geodesics are short if measured with the (Finsler) metric. Observe that $G L(\mathcal{H})^{s}$ is the union of the 
orbits of invertible selfadjoint operators. In particular, the orbit of the identity is $G L(\mathcal{H})^{+}$, the cone of positive invertible operators.

The study of the geometric structure of the set of selfadjoint operators, in the non-invertible case, was continued in [4], [5], [7] and [11]. The set $L(\mathcal{H})^{s}$ can be partitioned in certain classes, called Thompson components and on each component a complete metric can be defined. In the positive case, this metric is known as the Thompson metric, see [20]. It turns out that the component of a selfadjoint closed range operator admits a structure of a homogeneous space, see [4], [5] and [11]. In [7], the orbit $\mathcal{O}_{a}$ of a positive operator $a$ with closed range was studied, provided with a suitable metric $d$. It was proved that $\left(\mathcal{O}_{a}, d\right)$ is a fibre bundle and the orbit was described as the union of certain Thompson components. Also, the metric $d$ coincides with the operator norm on each component and the differential structure of each component is compatible with the fibre bundle structure of the orbit $\mathcal{O}_{a}$. Following this ideas, we study the differential structure of the orbit of a selfadjoint operator with closed range.

The article is organized as follows. Section 2 contains a brief survey on congruence and equivalence of operators. We also recall the notion of Thompson component of a selfadjoint operator, defined in [11]. In Section 3, we characterize the orbit of a positive operator as a union of certain Thompson components, more precisely, $\mathcal{O}_{a}=\bigcup_{u \in \mathcal{U}(\mathcal{H})} C_{u a u^{*}}$, where $\mathcal{U}(\mathcal{H})$ is the subgroup of $G L(\mathcal{H})$ of unitary operators. The orbit of a selfadjoint operator $a$ is related to the positive and negative parts of $a$ : if $a=a_{1}-a_{2}$ and $b=b_{1}-b_{2}$ (with $a_{i}, b_{i}$ positive and $\left.a_{1} a_{2}=b_{1} b_{2}=0\right)$ are the decompositions of $a$ and $b$ in their positive and negative parts, we prove that if $b_{i} \in \mathcal{O}_{a_{i}}, i=1,2$, and the nullspaces of $a$ and $b$ are unitarily equivalent, then $b \in \mathcal{O}_{a}$. Conversely, if $a=v_{a}|a|$ and $b=v_{b}|b|$ are the polar decompositions of $a, b \in L(\mathcal{H})^{s}$, we show that if $b \in \mathcal{O}_{a}$ then there exists a unitary operator $u$ such that $v_{b}=u v_{a} u^{*}$. In particular this condition is also necessary when $a$ has closed range. In fact, in this case, the orbit of $a$ is characterized by the dimension of the nullspace of $a$ and the dimensions of the ranges of $a_{i}, i=1,2$. Finally, in Section 4 we study the differential structure of the orbit of a selfadjoint operator $a$ with closed range. We introduce the following metric on the orbit of $a$ : $d(b, c)=\left(\|b-c\|^{2}+\left\|p_{b}-p_{c}\right\|^{2}\right)^{1 / 2}$, for $b, c \in \mathcal{O}_{a}$, where $p_{b}$ and $p_{c}$ are the orthogonal projections onto the ranges of $b$ and $c$, respectively. (In fact, we consider the same metric as in the positive case). With this new topology, $\left(\mathcal{O}_{a}, \mathrm{~d}\right)$ has a structure of differential manifold; moreover $\left(G L(\mathcal{H}), \mathcal{O}_{a}, \pi_{a}\right)$ is a principal fibre bundle, where $\pi_{a}(g)=L_{g} a$, for $g \in G L(\mathcal{H})$. This structure is compatible with the structure of homogeneous space of the Thompson components involved, because the metric $d$ coincides with the usual metric on each component. In fact, the fibre of $b \in \mathcal{O}_{a}$, by the map $\alpha: b \rightarrow v_{b}$, is the Thompson component of $b$. Even if the map $\alpha$ is not necessarily continuous with the operator norm (see for example [7]), it is continuous if the metric $d$ is considered in $\mathcal{O}_{a}$ and $\left(\mathcal{O}_{a}, \mathcal{U} \mathcal{O}_{v_{a}}, \alpha\right)$ is a fibre bundle, where $\mathcal{U O}_{v_{a}}$ is the unitary orbit of $v_{a}$. 


\section{Preliminaries}

Let $L(\mathcal{H})$ be the algebra of linear bounded operators of a separable Hilbert space $\mathcal{H}$. Throughout this paper we consider the following subsets of $L(\mathcal{H})$ : the set $L(\mathcal{H})^{s}$ of selfadjoint operators, the cone $L(\mathcal{H})^{+}$of positive operators and the set $C R(\mathcal{H})$ of closed range operators. Let $\mathcal{I}$ be the subset of $C R(\mathcal{H})$ of partial isometries, i.e., $\mathcal{I}=$ $\left\{v \in L(\mathcal{H}): v v^{*}\right.$ is an orthogonal projection $\}$. If $G L(\mathcal{H})$ is the group of invertible operators of $L(\mathcal{H})$ and $\mathcal{U}(\mathcal{H})$ the subgroup of $G L(\mathcal{H})$ of unitary operators, the set of reflections is $\mathcal{P}=\left\{v \in L(\mathcal{H}): v=v^{-1}=v^{*}\right\}$. If $\mathcal{A} \subseteq L(\mathcal{H})$, let $\mathcal{A}^{s}=\mathcal{A} \cap L(\mathcal{H})^{s}$.

Given $a \in L(\mathcal{H}), R(a)$ denotes the range of $a, N(a)$ its nullspace, $p_{a}$ denotes the orthogonal projection onto $\overline{R(a)}$ and $a^{\dagger}$ the Moore-Penrose pseudoinverse of $a$. If $\mathcal{S}$ is a closed subspace of $\mathcal{H}, p_{\mathcal{S}}$ denotes the orthogonal projection onto $\mathcal{S}$.

If $a \in L(\mathcal{H})$, we fix the following polar decomposition of $a: a=v_{a}|a|$ where $|a|=\left(a^{*} a\right)^{1 / 2}$ is positive and $v_{a}$ is a partial isometry from $N(a)^{\perp}$ onto $\overline{R(a)}$ with $N\left(v_{a}\right)=N(a)$. If $a$ is selfadjoint, the isometric part of the polar decomposition can be defined to obtain a reflection: in this case $R(a)^{\perp}=N(a)$ so that $u_{a}=$ $v_{a}+p_{N(a)} \in \mathcal{P}$ and $a=u_{a}|a|=|a| u_{a}$. Also, every $a \in L(\mathcal{H})^{s}$ admits a unique positive orthogonal decomposition (p.o.d.) as $a=a_{1}-a_{2}$, where $a_{1}, a_{2} \in L(\mathcal{H})^{+}$ and $a_{1} a_{2}=0$. In fact, it is easy to see that $a_{1}=\frac{|a|+a}{2}$ and $a_{2}=\frac{|a|-a}{2}$. The operator $a_{1}$ is called the positive part of $a$, and $-a_{2}$, the negative part.

\subsection{Equivalence and congruence of operators}

The following definitions and results about equivalence of operator ranges and equivalence and congruence of operators can be found in [10].

Two operator ranges $\mathcal{R}$ and $\mathcal{S}$ are similar if there exists $g \in G L(\mathcal{H})$ such that $\mathcal{R}=g(\mathcal{S})$ and unitarily equivalent if $g$ can be taken to be unitary. Operator ranges are similar if and only if they are unitarily equivalent.

Two operators $a, b \in L(\mathcal{H})$ are equivalent if there exist $g, f \in G L(\mathcal{H})$ such that $b=g a f$; the operators $a$ and $b$ are congruent if there exists $g \in G L(\mathcal{H})$ such that $b=g a g^{*}$.

Proposition 2.1. Normal operators are equivalent if and only if their ranges are unitarily equivalent.

Theorem 2.2. Let $a, b \in L(\mathcal{H})^{+}$, then the following conditions are equivalent:

1. $a$ and $b$ are equivalent,

2. $a^{1 / 2}$ and $b^{1 / 2}$ are equivalent,

3. $a$ and $b$ are congruent.

Remark 2.3. From Theorem 2.2, it follows that the ranges of two positive operators $a$ and $b$ are unitarily equivalent if and only if the ranges of $a^{1 / 2}$ and $b^{1 / 2}$ are unitarily equivalent. Also, it can be proved that if $a, b \in L(\mathcal{H})$, then $a$ and $b$ are equivalent if and only if $a^{*} a$ and $b^{*} b$ are congruent; or, equivalently, if $|a|$ and $|b|$ are congruent. By Theorem 2.2, two positive operators are equivalent if and only if 
they are congruent. This is no longer true for selfadjoint operators: for example if $a \in L(\mathcal{H})^{s}$ is not positive then $a$ and $|a|$ are equivalent but they are not congruent.

The following result about unitary congruence of projectors, is due to B. Sz.-Nagy, see [16], [17]. An alternative proof can be found in [14].

Proposition 2.4. If $p, q$ are two orthogonal projections satisfying that $\|p-q\|<1$ then there exists $u \in \mathcal{U}(\mathcal{H})$ such that $q=u p u^{*}$.

Remark 2.5. In [2], J. Avron, R. Seiler and B. Simon proved that given two orthogonal projections $p, q$ with $\|p-q\|<1$, then there exists $v \in \mathcal{U}(\mathcal{H})$ such that $q=v p v^{*}$ and $p=v q v^{*}$.

\subsection{Thompson components of selfadjoint operators}

Consider the following equivalence relation in $L(\mathcal{H})^{+}$: given $a, b \in L(\mathcal{H})^{+}, a \sim b$ if and only if there exist positive numbers $\alpha, \beta$ such that $a \leq \alpha b$ and $b \leq \beta a$, where $\leq$ is the partial order induced in $L(\mathcal{H})$ by $L(\mathcal{H})^{+}$. Therefore, $L(\mathcal{H})^{+}$is the (disjoint) union of equivalence classes, called Thompson components. Each component admits a complete metric, known as the Thompson part metric. Given $a \in L(\mathcal{H})^{+}$, it holds that $b \in C_{a}$ if and only if $R\left(b^{1 / 2}\right)=R\left(a^{1 / 2}\right)$. The reader is referred to [4] and [5] to find a complete exposition about the Thompson components of positive operators.

In [11], the notion of Thompson component is extended to selfadjoint operators. Consider a reflection $v \in \mathcal{P}$, then the indefinite sesquilinear form given by

$$
\langle x, y\rangle_{v}=\langle v x, y\rangle, \quad x, y \in \mathcal{H},
$$

defines an order in $L(\mathcal{H})$ : if $a, b \in L(\mathcal{H})$, then $a \leq_{v} b$ if and only if $v(b-a) \geq 0$.

Given $a, b \in L(\mathcal{H})^{s}$, with polar decompositions $a=u_{a}|a|$ and $b=u_{b}|b|, a \sim b$ if there exist $\alpha, \beta>0$ such that $a \leq_{u_{a}} \alpha b$ and $b \leq_{u_{b}} \beta b$. It follows that $\sim$ is an equivalence relationship. The Thompson component of $a$ is the set $C_{a}=\{b \in$ $L(\mathcal{H})^{s}: R\left(|b|^{1 / 2}\right)=R\left(|a|^{1 / 2}\right)$ and $\left.u_{b}=u_{a}\right\}$. In particular, if $a$ has closed range, then $C_{a}=\left\{b \in C R(\mathcal{H})^{s}: v_{b}=v_{a}\right\}$.

The component of $a \in L(\mathcal{H})^{s}$ is homeomorphic to the product of two Thompson components of positive operators, namely, $C_{a} \simeq C_{a_{1}} \times C_{a_{2}}$, where $a=a_{1}-a_{2}$ is the p.o.d. of $a$, see Theorem 5.1 of [11].

\section{The orbit of a selfadjoint operator}

In this section we study the orbit of congruence of a fixed selfadjoint operator $a$, i.e., the set of operators in $L(\mathcal{H})$ which are congruent to $a$. This set was characterized in the invertible case: two invertible selfadjoint operators are congruent if and only if the ranges of their positive and negative parts have the same dimension; or equivalently, the reflections of their polar decompositions are unitarily equivalent, see [11]. 
Consider the following action of $G L(\mathcal{H})$ over $L(\mathcal{H})^{s}$,

$$
L: G L(\mathcal{H}) \times L(\mathcal{H})^{s} \rightarrow L(\mathcal{H})^{s}, L_{g} a=g g^{*}, \quad a \in L(\mathcal{H})^{s}, g \in G L(\mathcal{H}) .
$$

Given $a \in L(\mathcal{H})^{s}$, the orbit of $a$ corresponding to the action $L$ is the set $\mathcal{O}_{a}$ of operators which are congruent with $a$, i.e.

$$
\mathcal{O}_{a}=\left\{g a g^{*}: g \in G L(\mathcal{H})\right\}
$$

The next proposition is a rewriting of Proposition 2.1 and Theorem 2.2, and provides a characterization of $\mathcal{O}_{a}$, when $a$ is positive.

Proposition 3.1. Consider $a, b \in L(\mathcal{H})^{+}$; then the following conditions are equivalent:

1. $b \in \mathcal{O}_{a}$,

2. $R(a)$ and $R(b)$ are unitarily equivalent,

3. $R\left(a^{1 / 2}\right)$ and $R\left(b^{1 / 2}\right)$ are unitarily equivalent.

Proof. 1 $\rightarrow 2$ : Follows from Proposition 2.1.

$2 \rightarrow 3$ : Follows from Remark 2.3 .

$3 \rightarrow 1$ : Suppose $R\left(b^{1 / 2}\right)=v R\left(a^{1 / 2}\right)$ for some $v \in \mathcal{U}(\mathcal{H})$, then, by Proposition $2.1, a^{1 / 2}$ and $b^{1 / 2}$ are equivalent. From Theorem 2.2, it follows that $a$ and $b$ are congruent, or equivalently, $b \in \mathcal{O}_{a}$.

As a consequence of Proposition 3.1 we have the following results.

Corollary 3.2. Consider $a, b \in L(\mathcal{H})^{+}$, then $b \in \mathcal{O}_{a}$ if and only if $b^{1 / 2} \in \mathcal{O}_{a^{1 / 2}}$.

Proposition 3.3. Consider $a, b \in L(\mathcal{H})^{+}$. If $b \in \mathcal{O}_{a}$ then $b^{t} \in \mathcal{O}_{a^{t}}$ for $t \in[0,1]$.

Proof. To prove this assertion we use that given $c, d \in L(\mathcal{H})^{+}$such that if $R(c)=$ $R(d)$ then $R\left(c^{t}\right)=R\left(d^{t}\right)$, for $t \in[0,1]$. In fact, if $R(c) \subseteq R(d)$ then, by Douglas Theorem (see [9]), there exists a constant $\lambda>0$ such that $c^{2} \leq \lambda^{2} d^{2}$. By the Löwner-Heinz inequality, (see [19]), $x^{t}$ is operator monotone increasing for $t \in[0,1]$, then $c^{2 t} \leq \lambda^{2 t} d^{2 t}$, so that $R\left(c^{t}\right) \subseteq R\left(d^{t}\right)$. The other inclusion follows similarly.

Suppose that $b \in \mathcal{O}_{a}$, then, by Proposition 3.1, there exists $u \in \mathcal{U}(\mathcal{H})$ such that $R(b)=R\left(u a u^{*}\right)$. Therefore, $R\left(b^{t}\right)=R\left(\left(u a u^{*}\right)^{t}\right)$, for any $t \in[0,1]$. Finally, applying Stone-Weierstrass theorem, it holds that $\left(u a u^{*}\right)^{t}=u a^{t} u^{*}$, so that $R\left(b^{t}\right)=R\left(u a^{t} u^{*}\right)$, or equivalently, $b^{t} \in \mathcal{O}_{a^{t}}$.

In particular, if $a \in C R(\mathcal{H})^{+}$, then $\mathcal{O}_{a}=\mathcal{O}_{a^{t}}$ for $t \in[0,1]$. In fact, by Theorem 2.7 of [5], $R(a)=R\left(a^{t}\right)$. If $b \in \mathcal{O}_{a}$ then $R(b)=u R(a)=u R\left(a^{t}\right)$, so that $b \in \mathcal{O}_{a^{t}}$. Hence $\mathcal{O}_{a}=\mathcal{O}_{a^{t}}$. More generally, it holds that if $b \in \mathcal{O}_{a}$ then $f(b) \in \mathcal{O}_{f(a)}$ for every $f \in \mathcal{C}(X)$ monotone increasing, with $X$ a compact set wich contains the spectrum of $a$.

The orbit of $a$ can be seen as a union of Thompson components, as shows the next corollary. 
Corollary 3.4. If $a \in L(\mathcal{H})^{+}$, then $\mathcal{O}_{a}=\bigcup_{u \in \mathcal{U}(\mathcal{H})} C_{\text {uau }}$, where $C_{\text {uau }}$ is the Thompson component of uau*, for $u \in \mathcal{U}(\mathcal{H})$.

In what follows we study the orbit of a selfadjoint operator.

Proposition 3.5. Consider $a, b \in L(\mathcal{H})^{s}$. If $b \in \mathcal{O}_{a}$, then $|b| \in \mathcal{O}_{|a|}$.

Proof. If $b \in \mathcal{O}_{a}$, then $a$ and $b$ are equivalent. By Remark 2.3, $|a|$ and $|b|$ are congruent, or equivalently, $|b| \in \mathcal{O}_{|a|}$.

Proposition 3.6. Consider $a, b \in L(\mathcal{H})^{s}$ with p.o.d. $a=a_{1}-a_{2}$ and $b=b_{1}-b_{2}$. If $b_{i} \in \mathcal{O}_{a_{i}}$ for $i=1,2$, and $N(b)$ is unitarily equivalent to $N(a)$, then $b \in \mathcal{O}_{a}$.

Proof. Since $N(b)$ and $N(a)$ are unitarily equivalent, there exists $u \in \mathcal{U}(\mathcal{H})$ such that $N(b)=u N(a)$. By Proposition 3.1, there exist $u_{1}, u_{2} \in \mathcal{U}(\mathcal{H})$ such that $R\left(b_{i}^{1 / 2}\right)=u_{i} R\left(a_{i}^{1 / 2}\right)$, for $i=1,2$. Then $b_{i}^{1 / 2}$ and $u_{i} a_{i}^{1 / 2} u_{i}^{*}$ have the same range and nullspace, so that (see Corollary 1, [10]) there exists $g_{i} \in G L(\mathcal{H})$ such that $b_{i}^{1 / 2}=g_{i} u_{i} a_{i}^{1 / 2} u_{i}^{*}$, or $b_{i}^{1 / 2} u_{i}=g_{i} u_{i} a_{i}^{1 / 2}, i=1,2$. Consider $w=g_{1} u_{1} p_{a_{1}}+g_{2} u_{2} p_{a_{2}}+$ $u\left(1-p_{a}\right)$. Then $w \in G L(\mathcal{H})$, in fact, it is easy to see that $w^{-1}=u_{1}^{*} g_{1}^{-1} p_{b_{1}}+$ $u_{2}^{*} g_{2}^{-1} p_{b_{2}}+u^{*}\left(1-p_{b}\right)$. On the other hand, $w a w^{*}=w\left(a_{1}-a_{2}\right) w^{*}=b_{1}^{1 / 2} u_{1} u_{1}^{*} b_{1}^{1 / 2}-$ $b_{2}^{1 / 2} u_{2} u_{2}^{*} b_{2}^{1 / 2}=b$. Hence $b \in \mathcal{O}_{a}$.

Given $v \in \mathcal{I}^{s}$, denote by $\mathcal{U O}_{v}$ the unitary orbit of $v$, i.e., the set $\mathcal{U} \mathcal{O}_{v}=$ $\left\{u v u^{*}: u \in \mathcal{U}(\mathcal{H})\right\}$. The next theorem relates the orbit of $a$ with the unitary orbits of $u_{a}$ and $v_{a}$, where $a \in L(\mathcal{H})^{s}$ has polar decomposition $a=u_{a}|a|=v_{a}|a|$ with $u_{a} \in \mathcal{P}$ and $v_{a}$ the partial isometry.

Theorem 3.7. Consider $a, b \in L(\mathcal{H})^{s}$. If $b \in \mathcal{O}_{a}$ then

1. $u_{b} \in \mathcal{U O}_{u_{a}}$,

2. $v_{b} \in \mathcal{U O}_{v_{a}}$.

In order to prove this theorem we need the following lemma, which is similar to a result proved by S. Hassi, Z. Sebestyen and S. V. De Snoo, see [13]. We include a proof which follows the ideas of the proof given by J. Antezana.

Lemma 3.8. Let $a, b \in L(\mathcal{H})^{+}$and $h, g \in L(\mathcal{H})$ be operators such that $b h=g a$, then there exists a unique $s \in L(\mathcal{H})$ such that

$$
b^{1 / 2} s=g a^{1 / 2} \text { and } N(b) \subseteq N\left(s^{*}\right) .
$$

Moreover, if $h, g \in G L(\mathcal{H})$ then there exists $s^{\prime} \in G L(\mathcal{H})$ such that

$$
b^{1 / 2} s^{\prime}=g a^{1 / 2} .
$$

Proof. It is sufficient to prove the existence of $s \in L(\mathcal{H})$ when $g$ is a contraction. If $b h=g a$, then

$$
g a^{2} g^{*}=b h h^{*} b \leq\|h\|^{2} b^{2} .
$$

Since $f(t)=t^{2}$ is operator convex and $\mathrm{f}(0)=0$, then, by Jensen's inequality (see [12]), it follows that $\left(g a g^{*}\right)^{2} \leq g a^{2} g^{*}$. Therefore $g a g^{*} \leq\left(g a^{2} g^{*}\right)^{1 / 2} \leq\|h\| b$, 
because $g(t)=t^{1 / 2}$ is operator monotone increasing. Hence, by Douglas Theorem (see [9]), there exists $s \in L(\mathcal{H})$ such that $b^{1 / 2} s=g a^{1 / 2}$. The uniqueness of $s$ follows from the fact that $N(b) \subseteq N\left(s^{*}\right)$.

Finally, if $g, h \in G L(\mathcal{H})$, then $h^{*} R(b)=R(a)$. Therefore, there exists $u \in$ $\mathcal{U}(\mathcal{H})$ such that $u R(b)=R(a)$ so that $u N(b)=N(a)$. Since $g^{-1} b=a h^{-1}$, then there exists a unique $l \in L(\mathcal{H})$ such that $g^{-1} b^{1 / 2}=a^{1 / 2} l$. Therefore

$$
l^{*} s^{*}\left(b^{1 / 2} x\right)=l^{*} a^{1 / 2} g^{*} x=b^{1 / 2}\left(g^{*}\right)^{-1} g^{*} x=b^{1 / 2} x,
$$

so that $l^{*} s^{*} p_{b}=p_{b}$. If we consider $s^{* \prime}=s^{*} p_{b}+u p_{N(b)}$ and $l^{* \prime}=l^{*} p_{a}+u^{*} p_{N(a)}$, it follows that $l^{* \prime} s^{* \prime}=l^{*} s^{*} p_{b}+u^{*} u p_{N(b)}=1$. Similarly, $s^{* \prime} l^{* \prime}=1$ and $s^{* \prime} \in G L(\mathcal{H})$. Also, it holds that $s^{* \prime} b^{1 / 2}=a^{1 / 2} g^{*}$.

Corollary 3.9. Let $a, b \in L(\mathcal{H})^{+}$and $g \in L(\mathcal{H})$ be operators such that $b^{\dagger}$ ga is well defined and bounded, then $\left(b^{1 / 2}\right)^{\dagger} g a^{1 / 2} \in L(\mathcal{H})$.

Proof. If $b^{\dagger} g a=h \in L(\mathcal{H})$ then $R\left(p_{b} g a\right) \subseteq R(b)$ and $b b^{\dagger} g a=b b^{\dagger} p_{b} g a=b h$ so that $p_{b} g a=b h$ because $b b^{\dagger} x=x$ for every $x \in R(b)$. Then, by Lemma 3.8, there exists a unique $s \in L(\mathcal{H})$ such that $b^{1 / 2} s=p_{b} g a^{1 / 2}$ and $N(b) \subseteq N\left(s^{*}\right)$. Therefore $p_{b} s=\left(b^{1 / 2}\right)^{\dagger} p_{b} g a^{1 / 2}=\left(b^{1 / 2}\right)^{\dagger} g a^{1 / 2}$. Since $R(s) \subseteq \overline{R(b)}$, it follows that $s=\left(b^{1 / 2}\right)^{\dagger} g a^{1 / 2}$.

Proof. (of Theorem 3.7) 1. Let $a=\alpha u_{a}$ and $b=\beta u_{b}$ be the polar decompositions of $a$ and $b$. Observe that $\alpha$ and $u_{a}$ commute so that $\alpha^{1 / 2}$ and $u_{a}$ commute, and the same holds for $\beta$ and $u_{b}$. Since $b \in \mathcal{O}_{a}$, there exists $g \in G L(\mathcal{H})$ such that $b=g a g^{*}$. Therefore, $\beta u_{b}=g \alpha u_{a} g^{*}$ or $\beta u_{b} g^{*-1} u_{a}=g \alpha$ so that $\beta^{\dagger} g \alpha \in L(\mathcal{H})$. Applying Corollary 3.9, if $s=\left(\beta^{1 / 2}\right)^{\dagger} g \alpha^{1 / 2} \in L(\mathcal{H})$ then $v_{b} \beta^{1 / 2}=s v_{a} \alpha^{1 / 2} g^{*}$, so that $\beta^{1 / 2} v_{b}=g \alpha^{1 / 2} v_{a} s^{*}$ and then, $v_{b}=s v_{a} s^{*}$. Since $b=g a g^{*}$, then $N(a)=$ $g^{*} N(b)$, so that there exists $u \in \mathcal{U}(\mathcal{H})$ such that $N(b)=u N(a)$. Observe that $s=p_{b} s p_{a}$. Consider $s^{\prime}=s p_{a}+u\left(1-p_{a}\right)$, then $s^{\prime} \in G L(\mathcal{H})$ (see Lemma 3.8) and $s^{\prime} u_{a} s^{\prime *}=s v_{a} s^{*} p_{b}+u\left(1-p_{a}\right) u^{*}\left(1-p_{b}\right)=u_{b}$. Finally, if $s^{\prime}=w\left|s^{\prime}\right|$ is the polar decomposition of $s^{\prime}$, with $w$ unitary, it follows easily that $w u_{a} w^{*}=u_{b}$, or $u_{b} \in \mathcal{U} \mathcal{O}_{u_{a}}$.

2. Consider $w \in \mathcal{U}(\mathcal{H})$ as in the proof of 1 . Then $w$ also satisfies that $w v_{a} w^{*}=$ $v_{b}$.

Remark 3.10. Consider $a, b \in L(\mathcal{H})^{s}$ with positive orthogonal decompositions $a=$ $a_{1}-a_{2}$ and $b=b_{1}-b_{2}$. If $b \in \mathcal{O}_{a}$, it follows from from Theorem 3.7, that $p_{b} \in \mathcal{U} \mathcal{O}_{p_{a}}$ and $p_{a_{i}} \in \mathcal{U O}_{p_{a_{i}}}, i=1,2$.

When $a \in L(\mathcal{H})^{s}$ has closed range, it is possible to give a more accurate characterization of $\mathcal{O}_{a}$ as show the following results.

Theorem 3.11. Consider $a, b \in C R(\mathcal{H})^{s}$. Then $b \in \mathcal{O}_{a}$ if and only if $v_{b} \in \mathcal{U O}_{v_{a}}$. 
Proof. If $v_{b} \in \mathcal{U O}_{v_{a}}$ then there exists $u \in \mathcal{U}(\mathcal{H})$ such that $v_{b}=u v_{a} u^{*}$. Then $v_{b}=u p_{a_{1}} u^{*}-u p_{a_{2}} u^{*}$ is the p.o.d. of $v_{b}$, so that $p_{b_{i}}=u p_{a_{i}} u^{*}$, for $i=1,2$, and $p_{b}=p_{b_{1}}+p_{b_{2}}=u p_{a} u^{*}$. Then, by Proposition 3.6, $b \in \mathcal{O}_{a}$. The converse follows by Theorem 3.7.

For $n, m_{1}, m_{2} \in \mathbb{N} \cup\{0, \infty\}$ such that $n+m_{1}+m_{2}=\operatorname{dim} \mathcal{H}$, define

$$
\begin{aligned}
\mathcal{A}_{n, m_{1}, m_{2}}= & \left\{b \in C R(\mathcal{H})^{s}: \operatorname{dim} N(b)=n, \operatorname{dim} R\left(b_{i}\right)=m_{i}, i=1,2,\right. \\
& \text { where } \left.b=b_{1}-b_{2} \text { is the p.o.d. of } b\right\} .
\end{aligned}
$$

Proposition 3.12. If $a \in \mathcal{A}_{n, m_{1}, m_{2}}$, then $\mathcal{O}_{a}=\mathcal{A}_{n, m_{1}, m_{2}}$.

Proof. Let $a=a_{1}-a_{2}$ be the p.o.d. of $a$. If $b \in \mathcal{O}_{a}$ then $p_{b} \in \mathcal{U} \mathcal{O}_{p_{a}}$ and $p_{b_{i}} \in$ $\mathcal{U O}_{p_{a_{i}}}, i=1,2$, see Remark 3.10. Then, $\operatorname{dim} N(b)=\operatorname{dim} N(a)=n, \operatorname{dim} R\left(b_{i}\right)=$ $\operatorname{dim} R\left(a_{i}\right)=m_{i}, i=1,2$, so that $b \in \mathcal{A}_{n, m_{1}, m_{2}}$.

Conversely, consider $b \in \mathcal{A}_{n, m_{1}, m_{2}}$. Then, since $\mathcal{H}=R\left(a_{1}\right) \oplus R\left(a_{2}\right) \oplus N(a)=$ $R\left(b_{1}\right) \oplus R\left(b_{2}\right) \oplus N(b)$, there exists $u \in \mathcal{U}(\mathcal{H})$ such that $u N(a)=N(b)$ and $u R\left(a_{i}\right)=$ $R\left(b_{i}\right), i=1,2$. If $q=u p_{a} u^{*}$ then $q^{2}=q=q^{*}$ and $R(q)=u R(a)=R(b)$ so that $q=p_{b}$. Then $p_{b} \in \mathcal{U O}_{p_{a}}$. Similarly, it holds that $p_{b_{i}} \in \mathcal{U O}_{p_{a_{i}}}, i=1,2$. Then, by Proposition 3.6, it follows that $b \in \mathcal{O}_{a}$.

Corollary 3.13. Let $\mathcal{B}_{n, m_{1}, m_{2}}=\mathcal{I}^{s} \cap \mathcal{A}_{n, m_{1}, m_{2}}$. If $v \in \mathcal{B}_{n, m_{1}, m_{2}}$, then $\mathcal{U} \mathcal{O}_{v}=$ $\mathcal{B}_{n, m_{1}, m_{2}}$.

The orbit of $a \in C R(\mathcal{H})^{s}$ can also be related to the orbits of the positive and the negative parts of $a$.

Proposition 3.14. Let $a \in C R(\mathcal{H})^{s}$ with p.o.d. $a=a_{1}-a_{2}$, then

$$
\mathcal{O}_{a}=\left\{b_{1}-b_{2}: b_{i} \in \mathcal{O}_{a_{i}}, i=1,2, p_{b_{1}}+p_{b_{2}} \in \mathcal{U} \mathcal{O}_{p_{a}}\right\} .
$$

Proof. Consider $b \in \mathcal{O}_{a}$ with p.o.d. $b=b_{1}-b_{2}$. By Remark 3.10, it holds that $p_{b}=p_{b_{1}}+p_{b_{2}} \in \mathcal{U O}_{p_{a}}$ and $p_{b_{i}} \in \mathcal{U} \mathcal{O}_{p_{a_{i}}}, i=1,2$. Then $\mathcal{O}_{p_{b_{i}}}=\mathcal{O}_{p_{a_{i}}}$, for $i=1,2$ so that, by Proposition 3.1, it follows that $\mathcal{O}_{a_{i}}=\mathcal{O}_{p_{a_{i}}}$ and $\mathcal{O}_{b_{i}}=\mathcal{O}_{p_{b_{i}}}$. Therefore $\mathcal{O}_{a_{i}}=\mathcal{O}_{b_{i}}, i=1,2$.

Conversely, consider $b=b_{1}-b_{2}$ such that $b_{i} \in \mathcal{O}_{a_{i}}, i=1,2$, and $p_{b_{1}}+p_{b_{2}} \in$ $\mathcal{U O}_{p_{a}}$. Therefore, there exists $u \in \mathcal{U}(\mathcal{H})$ such that $p_{b_{1}}+p_{b_{2}}=u p_{a} u^{*}$, so that $p_{b_{1}}+$ $p_{b_{2}}$ is an orthogonal projection. Then the ranges $R\left(b_{1}\right)$ and $R\left(b_{2}\right)$ are orthogonal, so that $b=b_{1}-b_{2}$ is the p.o.d. of $b$. Therefore $p_{b}=p_{b_{1}}+p_{b_{2}} \in \mathcal{U O}_{p_{a}}$. Since $b_{i} \in \mathcal{O}_{a_{i}}$ then $p_{b_{i}} \in \mathcal{U} \mathcal{O}_{p_{a_{i}}}$. Then, by Proposition 3.6, it follows that $b \in \mathcal{O}_{a}$.

\section{The differential geometry of the orbit of a closed range selfadjoint operator}

The purpose of this section is to study the differential structure of the set $\mathcal{O}_{a}$, when $a \in L(\mathcal{H})^{s}$ has closed range. Along this section we fix $a \in C R(\mathcal{H})^{s}$ with p.o.d $a=a_{1}-a_{2}$ and polar decomposition $a=|a| v_{a}$. 
Consider the following maps

$$
\pi_{a}: G L(\mathcal{H}) \rightarrow \mathcal{O}_{a}, \pi_{a}(g)=g a g^{*} \text { and } \alpha: \mathcal{O}_{a} \rightarrow \mathcal{U} \mathcal{O}_{v_{a}}, \alpha(b)=v_{b}
$$

Proposition 4.1. Let $a \in C R(\mathcal{H})^{s}$, then the fibre of $v_{a}$ by $\alpha$ is the Thompson component of a, i.e. $\alpha^{-1}\left(\left\{v_{a}\right\}\right)=C_{a}$.

Proof. Consider $b \in \alpha^{-1}\left(\left\{v_{a}\right\}\right)$, then $b \in \mathcal{O}_{a}$ and $v_{b}=v_{a}$. Therefore $b \in C_{a}$. Conversely, if $b \in C_{a}$ then $v_{b}=v_{a}$ and so $v_{b} \in \mathcal{U O}_{v_{a}}$. Hence, by Theorem 3.11, $b \in \mathcal{O}_{a}$. Since $v_{b}=v_{a}$ and $b \in \mathcal{O}_{a}$, it holds that $b \in \alpha^{-1}\left(\left\{v_{a}\right\}\right)$.

To provide $\mathcal{O}_{a}$ with a structure of differential manifold we need the mapping $\pi_{a}: G L(\mathcal{H}) \rightarrow \mathcal{O}_{a}, \pi_{a}(g)=g a g^{*}$, to have local cross sections. But this may not happen if we consider in $\mathcal{O}_{a}$ the topology given by the operator norm, see, for example, Theorem 3.4 of [7]. Consider the following metric in $\mathcal{O}_{a}$ :

$$
d(b, c)=\left(\|b-c\|^{2}+\left\|p_{b}-p_{c}\right\|^{2}\right)^{1 / 2} .
$$

Observe that $d$ coincides with the usual metric in each Thompson component. In [6], this metric was considered on the set $C R(\mathcal{H})$ and it was proved that the mapping $\mu:(C R(\mathcal{H}), d) \rightarrow(C R(\mathcal{H}),\|\cdot\|), \mu(b)=b^{\dagger}$ is continuous.

Proposition 4.2. The map $\alpha:\left(\mathcal{O}_{a}, d\right) \rightarrow\left(\mathcal{U} \mathcal{O}_{v_{a}},\|\|.\right), \alpha(b)=v_{b}$ is continuous.

Proof. The continuity of $\alpha$ follows from the facts that $v_{b}=b^{\dagger}|b|$ and the maps $\mu$ : $(C R(\mathcal{H}), d) \rightarrow\left(\mathcal{O}_{a},\|\|.\right), \mu(b)=b^{\dagger}$ and $||:.\left(\mathcal{O}_{a}, d\right) \rightarrow\left(L(\mathcal{H})^{+},\|\|.\right)$are continuous, see Theorem 4.2 of $[6]$.

Corollary 4.3. The map $p_{i}:\left(\mathcal{O}_{a}, d\right) \rightarrow\left(\mathcal{U O}_{p_{a_{i}}},\|\|.\right), p_{i}(b)=p_{b_{i}}$ is continuous, for $i=1,2$, where $b=b_{1}-b_{2}$ is the p.o.d. of $b$.

Proof. It holds that $p:\left(\mathcal{O}_{a}, d\right) \rightarrow\left(\mathcal{U O}_{p_{a}},\|\|.\right), p(b)=p_{b}=b b^{\dagger}$ is continuous. Therefore, by Proposition 4.2, it follows that $p_{1}:\left(\mathcal{O}_{a}, d\right) \rightarrow\left(\mathcal{U} \mathcal{O}_{p_{a_{1}}},\|\cdot\|\right)$ is continuous since $p_{1}(b)=p_{b_{1}}=\frac{v_{b}+p_{b}}{2}$. In the same way, $p_{2}$ is continuous.

In order to prove the existence of local cross sections of $\pi$, we recall the following result, see [14].

Lemma 4.4. Let $p, q \in L(\mathcal{H})$ be orthogonal projections such that $\|p-q\|<1$ and consider $h=1-(p-q)^{2}$. Then $h \in G L(\mathcal{H})^{+}$and $q h^{-1 / 2} p h^{-1 / 2} q=q$.

Proof. $h \in G L(\mathcal{H})^{+}$because $\|p-q\|<1$. It is easy to see that $p$ and $q$ commute with $h$; in fact, $h q=q h=q p q$. Then $p$ and $q$ also commute with $h^{-1 / 2}$ and $q h^{-1 / 2} p h^{-1 / 2} q=q p q h^{-1}=q$.

Theorem 4.5. The map $\pi_{a}:(G L(\mathcal{H}),\|\|.) \rightarrow\left(\mathcal{O}_{a}, d\right), \pi_{a}(g)=g a g^{*}$ is continuous and it admits continuous local cross sections. 
Proof. It holds that $p_{\text {gag }^{*}}$ depends continuously on $g \in G L(\mathcal{H})$, since the orthogonal projection onto $g(R(a))$ is given by the formula

$$
p_{g a g^{*}}=p_{g(R(a))}=g p_{a} g^{-1}\left(g p_{a} g^{-1}\right)^{*}\left(1-\left(g p_{a} g^{-1}-\left(g p_{a} g^{-1}\right)^{*}\right)^{2}\right)^{-1},
$$

see [1]. Also, gag* depends continuously on $g \in G L(\mathcal{H})$. Then, it follows that $\pi_{a}:(G L(\mathcal{H}),\|\|.) \rightarrow\left(\mathcal{O}_{a}, d\right)$ is continuous.

Let $p:\left(\mathcal{O}_{a}, d\right) \rightarrow\left(\mathcal{U O}_{p_{a}},\|\|.\right), p(b)=p_{b}$ and $p_{i}:\left(\mathcal{O}_{a}, d\right) \rightarrow\left(\mathcal{U O}_{p_{a_{i}}},\|\|.\right)$, $p_{i}(b)=p_{b_{i}}$. Since $p$ and $p_{i}$ are continuous there exists $\delta>0$ such that $\left\|p_{b}-p_{a}\right\|<$ $1,\left\|p_{b_{i}}-p_{a_{i}}\right\|<1, i=1,2$, if $b \in \mathcal{O}_{a}$ and $d(b, a)<\delta$. Consider $b \in \mathcal{O}_{a}$ such that $d(b, a)<\delta$, then $g=1-\left(p_{b}-p_{a}\right)^{2} \in G L(\mathcal{H})^{+}$and $g_{i}=1-\left(p_{b_{i}}-p_{a_{i}}\right)^{2} \in$ $G L(\mathcal{H})^{+}, i=1,2$.

Define $s(b)=b_{1}^{1 / 2} g_{1}^{-1 / 2}\left(a_{1}^{\dagger}\right)^{1 / 2}-b_{2}^{1 / 2} g_{2}^{-1 / 2}\left(a_{2}^{\dagger}\right)^{1 / 2}+\left(1-p_{b}\right) g^{-1 / 2}\left(1-p_{a}\right)$. It is easy to see, applying Lemma 4.4 , that $s(b)^{-1}=a_{1}^{1 / 2} g_{1}^{-1 / 2}\left(b_{1}^{\dagger}\right)^{1 / 2}-a_{2}^{1 / 2} g_{2}^{-1 / 2}\left(b_{2}^{\dagger}\right)^{1 / 2}+$ $\left(1-p_{a}\right) g^{-1 / 2}\left(1-p_{b}\right)$ so that $s(b) \in G L(\mathcal{H})$. Also, by Lemma 4.4 , it follows that $\pi(s(b))=b$. Then $s$ is a continuous local cross section of $\pi$ in a neighbourhood of $a$. If $c=g a g^{*}, g \in G L(\mathcal{H})$, consider $s^{\prime}=l_{g} \circ s \circ L_{g^{-1}}$, where $L_{g}: C R(\mathcal{H})^{s} \rightarrow \mathcal{O}_{a}, L_{g}(b)=g b g^{*}$ and $l_{g}: G L(\mathcal{H}) \rightarrow G L(\mathcal{H})$ is the left multiplication by $g$. It follows that $s^{\prime}$ is a local section of $\pi$ in a neighbourhood of $c$.

Observe that from the above proposition, it follows that $\mathcal{O}_{a}$ is open in $\left(L(\mathcal{H})^{s}, d\right)$, so that it is also closed. Denote by $I_{a}$ the isotropy group of $a$ by the action $L$, i.e. $I_{a}=\left\{g \in G L(\mathcal{H}): g a g^{*}=a\right\}$.

Corollary 4.6. Consider $a \in C R(\mathcal{H})^{s}$, then the metric space $\left(\mathcal{O}_{a}, d\right)$ is homeomorphic to the quotient space $G L(\mathcal{H}) / I_{a}$, where the quotient topology is considered.

It can be proved that $I_{a}$ is a regular subgroup of $G L(\mathcal{H})$, i.e., $I_{a}$ is a BanachLie group and $\left(T I_{a}\right)_{1}$ is a closed and complemented subspace of $(T G L(\mathcal{H}))_{1}$. Then it follows that $G L(\mathcal{H}) / I_{a}$ has a structure of a differential manifold and, by the above corollary, $\left(\mathcal{O}_{a}, d\right)$ is differentiable manifold. It also holds that $\left(G L(\mathcal{H}), \mathcal{O}_{a}, \pi_{a}\right)$ is a principal fibre bundle with structural group $I_{a}$, see [15]. Then it is possible to define a natural connection on $\mathcal{O}_{a}$ which induces the concept of parallel field along a curve so that the notion of geodesic is defined. Also given $X$ in the tangent space of $\mathcal{O}_{a}$ on $a$, there exists a unique geodesic $\gamma \subseteq \mathcal{O}_{a}$ such that $\gamma(0)=a$ and $\dot{\gamma}(0)=X$. The details follows as in the positive case, the reader can find them in [7]. Finally, given $b, c \in C_{a} \subseteq \mathcal{O}_{a}$, there exists a unique geodesic in $C_{a}$ joining $b$ and $c$, namely, $\gamma_{b, c}(t)=|b|^{1 / 2}\left\{\left(|b|^{1 / 2}\right)^{\dagger}|c|\left(|b|^{1 / 2}\right)^{\dagger}\right\}^{t}|b|^{1 / 2} v_{a}$, for $t \in[0,1]$, see [11].

In what follows we show that $\left(\mathcal{O}_{a}, \mathcal{U O}_{v_{a}}, \alpha\right)$ is a fibre bundle. We first prove a technical result.

Lemma 4.7. Consider $w \in \mathcal{I}^{s}$. There exists $0<\delta<1$ such that if $v \in \mathcal{I}^{s}$ and $\|v-w\|<\delta$ then $\left\|p_{v}-p_{w}\right\|<1$ and $\left\|p_{i}-q_{i}\right\|<1$, for $i=1,2$, where $v=p_{1}-p_{2}$ and $w=q_{1}-q_{2}$. 
Proof. Since $v \rightarrow v^{2}$ is continuous, there exists $\delta>0$ such that $\left\|p_{v}-p_{w}\right\|=\| v^{2}-$ $w^{2} \|<1$ if $\|v-w\|<\delta$. Consider $v \in \mathcal{I}^{s}$ with p.o.d. $v=p_{1}-p_{2}$. Since $p_{v}=p_{1}+p_{2}$ and $p_{w}=q_{1}+q_{2}$, then $\left\|p_{1}-q_{1}\right\|=\frac{1}{2}\left\|v-w+p_{v}-p_{w}\right\| \leq \frac{1}{2}\left(\|v-w\|+\left\|p_{v}-p_{w}\right\|\right)<1$ if $\|v-w\|<\delta$ for $\delta$ small enough. Similarly for $\left\|p_{2}-q_{2}\right\|$.

Remark 4.8. Consider $a \in C R(\mathcal{H})^{s}$ with polar decomposition $a=|a| v_{a}$. Observe that $V_{a}=\left\{v \in \mathcal{I}^{s}:\left\|v-v_{a}\right\|<\delta\right\} \subseteq \mathcal{U O}_{v_{a}}$, with $\delta$ as in Lemma 4.7. In fact, by Proposition 2.4 and Lemma 4.7, given $v \in V_{a}$ it follows that there exist $u_{1}, u_{2}, u \in$ $\mathcal{U}(\mathcal{H})$ such that $p_{i}=u_{i} p_{a_{i}} u_{i}^{*}$, for $i=1,2$ and $p_{v}=u p_{a} u^{*}$, where $v=p_{1}-p_{2}$. If $w=u_{1} p_{a_{1}}+u_{2} p_{a_{2}}+u\left(1-p_{a}\right)$ then it is easy to see that $w \in \mathcal{U}(\mathcal{H})$ and $w v_{a} w^{*}=v$, i.e. $v \in \mathcal{U O}_{v_{a}}$.

Proposition 4.9. For $b \in \mathcal{O}_{a}$ let $V_{b}=\left\{v \in \mathcal{I}^{s}:\left\|v-v_{b}\right\|<\delta\right\}$ with $\delta$ as in Lemma 4.7. Then the $d$-open set $\alpha^{-1}\left(V_{b}\right)$ is homeomorphic to the product $C_{b} \times V_{b}$, where $C_{b}$ is the Thompson component of $b$.

Proof. Consider $e_{i}=1-\left(p_{i}-p_{b_{i}}\right)^{2}, i=1,2$ and $e=1-\left(p_{v}-p_{b}\right)^{2}$, then $e_{i}, e \in$ $G L(\mathcal{H})^{+}, i=1,2$. Define $\phi(v)=p_{1} e_{1}^{-1 / 2} p_{b_{1}}+p_{2} e_{2}^{-1 / 2} p_{b_{2}}+\left(1-p_{v}\right) e^{-1 / 2}\left(1-p_{b}\right)$. Applying Lemma 4.4, it follows that $\phi(v) \in \mathcal{U}(\mathcal{H})$ and $\phi(v) v_{b} \phi(v)^{*}=v$.

Now define $f_{b}: C_{b} \times V_{b} \rightarrow \alpha^{-1}\left(V_{b}\right)$ such that $f_{b}(c, v)=\phi(v) c \phi(v)^{*}$. The map $f_{b}$ is well define. In fact, since $c \in C_{b} \subseteq \mathcal{O}_{b}=\mathcal{O}_{a}$ then $f_{b}(c, v)=\phi(v) c \phi(v)^{*} \in \mathcal{O}_{a}$. On the other hand, since $c \in C_{b}, \phi(v) \in \mathcal{U}(\mathcal{H})$ and $\phi(v) v_{b} \phi(v)^{*}=v$, then $f_{b}(c, v)=$ $\phi(v)|c| \phi(v)^{*} v$. Therefore, since $R(v)=\phi(v) R(b)=\phi(v) R(c)=R\left(f_{b}(c, v)\right)$, we get that $v_{f_{b}(c, v)}=v$. Then $\left\|v_{f_{b}(c, v)}-v_{b}\right\|=\left\|v-v_{b}\right\|<\delta$ since $v \in V_{b}$. Hence $f_{b}(c, v) \in \alpha^{-1}\left(V_{b}\right)$.

Consider now $h: \alpha^{-1}\left(V_{b}\right) \rightarrow C_{b} \times V_{b}$ such that $h(x)=\left(\phi\left(v_{x}\right)^{*} x \phi\left(v_{x}\right), v_{x}\right)$. The map $h$ is well defined. In fact, given $x \in \alpha^{-1}\left(V_{b}\right), \alpha(x)=v_{x} \in V_{b}$. Also, since $\phi\left(v_{x}\right)^{*} v_{x} \phi\left(v_{x}\right)=v_{b}$, it holds that $d=\phi\left(v_{x}\right)^{*} x \phi\left(v_{x}\right)=\phi\left(v_{x}\right)^{*}|x| \phi\left(v_{x}\right) v_{b}$. Then $v_{d}=v_{b}$ due to the fact that $R\left(v_{b}\right)=\phi\left(v_{x}\right)^{*} R(x)=R(d)$. Therefore, $d \in C_{b}$. Also, $\left(f_{b} \circ h\right)(x)=x$ and $\left(h \circ f_{b}\right)(c, v)=(c, v)$, due to the fact that $v_{f_{b}(c, v)}=v$.

Since $d(b, c)=\left(\|b-c\|^{2}+\left\|p_{b}-p_{c}\right\|^{2}\right)^{1 / 2}$ and $p_{f_{b}(c, v)}=p_{v}$; to see that $f_{b}$ is continuous is sufficient to prove that $\left(\mathcal{I}^{s},\|\|\right) \rightarrow(L(\mathcal{H}),\|\|), v \rightarrow \phi(v)$ is continuous. But, by Corollary 4.3 , the maps $\left(\mathcal{I}^{s},\|\|\right) \rightarrow(L(\mathcal{H}),\|\|), v \rightarrow p_{v}$ and $\left(\mathcal{I}^{s},\|\|\right) \rightarrow$ $(L(\mathcal{H}),\|\|), v \rightarrow p_{v_{i}}, i=1,2$ are continuous. Hence $f_{b}$ is continuous. By Proposition 4.2 and Corollary 4.3 ; it follows that the map $\left(\mathcal{O}_{a}, d\right) \rightarrow\left(\mathcal{U O} \mathcal{V}_{a},\|\cdot\|\right), x \rightarrow \phi\left(v_{x}\right)$ is continuous. Hence $h$ is continuous.

Corollary 4.10. Consider $a \in C R(\mathcal{H})^{s}$ then, $\left(\mathcal{O}_{a}, \mathcal{U} \mathcal{O}_{v_{a}}, \alpha\right)$ is a fibre bundle.

\section{Acknowledgment}

We are grateful to Professor Gustavo Corach for his helpful comments and suggestions. 


\section{References}

[1] E. Andruchow, G. Corach and D. Stojanoff, Geometry of oblique projections. Studia Math. 1371 (1999), 61-79.

[2] J. Avron, R. Seiler and B. Simon, The index of a pair of projections. J. Funct. Anal. 1201 (1994), 220-237.

[3] F. F. Bonsall and J. Duncan, Complete Normed Algebras. Springer-Verlag, 1973.

[4] G. Corach and A. Maestripieri, Differential and metrical structure of positive operators. Positivity 4 (1999), 297-315.

[5] G. Corach and A. Maestripieri, Differential geometry on Thompson's components of positive operators. Rep. Math. Phys. 45 (2000), 23-37.

[6] G. Corach, A. Maestripieri and M. Mbekhta, Metric and homogeneous structures of sets of closed range operators. J. of Operator Theory (2006), in press.

[7] G. Corach, A. Maestripieri and D. Stojanoff, Orbits of positive operators from a differentiable viewpoint. Positivity 8 (2004), 31-48.

[8] G. Corach, H. Porta and L. Recht, The geometry of spaces of selfadjoint invertible elements of a $C^{*}$-algebra. Integral Equations Operator Theory 16 (1993), 333-359. Operator Theory

[9] R. G. Douglas, On majorization, factorization and range inclusion of operators in Hilbert space. Proc. Amer. Math. Soc. 17 (1966), 413-416.

[10] P. A. Fillmore and J. P. Williams, On operator ranges. Adv. Math. 7 (1971), 254-281.

[11] G. Fongi and A. Maestripieri, Differential structure of the Thompson components of Selfadjoint Operators. Proc. Amer. Math. Soc. 136 (2008), 613-622.

[12] F. Hansen and G. K. Pedersen, Jensen's inequality for operators and Lowner's theorem. Math. Ann. 258 (1981/82), 229-241.

[13] S. Hassi, Z. Sebestyen and S. V. De Snoo, On the nonnegativity of operator products. Acta Math. Hungar. 109 1-2 (2005), 1-14.

[14] Y. Kato, An elementary proof of Sz.-Nagy theorem. Math. Japon. 20 (1975), 257-258.

[15] S. Lang, Differentiable Manifolds. Addison-Wesley, Reading, Mass., 1972.

[16] B. Nagy, Spektraldarstellung linearer Transformationen des Hilbertschen Raumes. Ergebnisse, d. Math. V/5 Berlin (1942).

[17] B. Nagy, Perturbations des transfomations autoajointes dans l'espace de Hilbert, Commentarii Math. Helv., 19 (1946/47), 347-266.

[18] R. Nussbaum, Hilbert's projective metric and iterated non linear maps. Memoirs Amer. Math. Soc. 391 (1988).

[19] G. K. Pedersen, Some operator monotone functions. Proc. Amer. Math. Soc. 36 (1972), 309-310.

[20] A. C. Thompson, On certain contraction mappings in a partially ordered vector space. Proc. Amer. Math. Soc. 14 (1963), 438-443. 
Guillermina Fongi

Instituto Argentino de Matemática

Saavedra $153 \mathrm{p}$

Capital Federal

Argentina

e-mail: gfongi@dm.uba.ar

Alejandra Maestripieri

Facultad de Ingeniería

Paseo Colón 850

Capital Federal

Argentina

e-mail: amaestri@fi.uba.ar 